\title{
Development of a Method to Characterize Active Sites in Photocatalysis using operando Transmission Electron Microscopy
}

\author{
Noah Glachman, ${ }^{1}$ Noah Geller, ${ }^{1}$ Alexander Shea, ${ }^{1}$ V. Antoine Verret, ${ }^{1}$ Khim Karki, ${ }^{2}$ Julio Rodriquez- \\ Manzo, ${ }^{2}$ Norman J. Salmon, ${ }^{2}$ Daan Hein Alsem, ${ }^{2}$ Deep Jariwala, ${ }^{3}$ and Eric Stach $^{1}$ \\ 1. Department of Materials Science and Engineering, University of Pennsylvania, Philadelphia, PA \\ 19104 \\ 2. Hummingbird Scientific, Lacey, WA \\ 3. Department of Electrical and Systems Engineering, University of Pennsylvania, Philadelphia, PA \\ 19104 \\ * Corresponding author: stach@seas.upenn.edu
}

Hydrogen gas has the potential to be a clean source of sustainable energy due to its high energy density. However, greenhouse gas emissions are still a major byproduct of current hydrogen production methods. Photoelectrochemistry provides a promising, environmentally friendly route to hydrogen production; [1,2] however, the atomic scale mechanisms of the photocatalysts that facilitate the water splitting reaction are currently poorly understood. Further understanding of the chemical physics governing the active hydrogen evolution sites would allow for better design of photoelectrochemical devices and thus lead to improved reaction efficiencies. This will overcome one of the major barriers impeding this promising technology.

We have developed a unique operando photoelectrochemistry transmission electron microscope (TEM) liquid cell sample holder which can be used to characterize these reactions in real time at nanometer length scales. This system builds upon our prior developments of operando electrochemical liquid cell holders $[3,4]$, by including the additional provision of an optical fiber directed at the sample to provide full solar spectrum illumination.

In order to provide accurate, quantitative information, it is necessary to accurately deposit the photocatalyst of interest onto microfabricated electrodes. In this research, a precise sample deposition technique utilizing an inkjet printer has been developed along with stable suspensions of known photocatalysts, leading to site-specific deposition onto the electrode chips. Specifically, this experimental design allows for correlation between I-V characteristics (Figure 1) and real time, high magnification imaging and spectroscopy, elucidating information about photocatalytic mechanisms at the nanoscale. The following photocatalysts used were chosen because the proposed mechanism for each exhibits a spatial dependence: plasmonically enhanced catalysis for Au nanoprisms and catalytically active edge sites for $\mathrm{MoS}_{2}$ flakes. These experiments will lay the groundwork for the use of this novel experimental design to investigate a wide variety of photoelectrochemical systems, and will allow determination of the mechanisms by which selected photocatalysts induce water splitting and the identification of defect features that serve as the active sites.

\section{References:}

[1] Nowotny, J.; Sorrell, C. C.; Sheppard, L. R.; Bak, T. Int. J. Hydrogen Energy 2005, 30, 521

[2] Walter, M. G. et al. Solar water splitting cells. Chem. Rev. 110, 6446-6473 (2010).

[3] N. Singh, T.S. Arthur, T.S, O. Tutusaus, J. Li, K. Kisslinger, H.L. Xin, E.A. Stach, X. Fan, and R.Mohtadi, ACS Appl. Energy Mat. 1, 4651-4661, 2018 
[4] Funding for the development of the sample holder was provided by the Department of Energy, Office of Basic Energy Sciences, through SBIR Grant \# DE-SC0015213
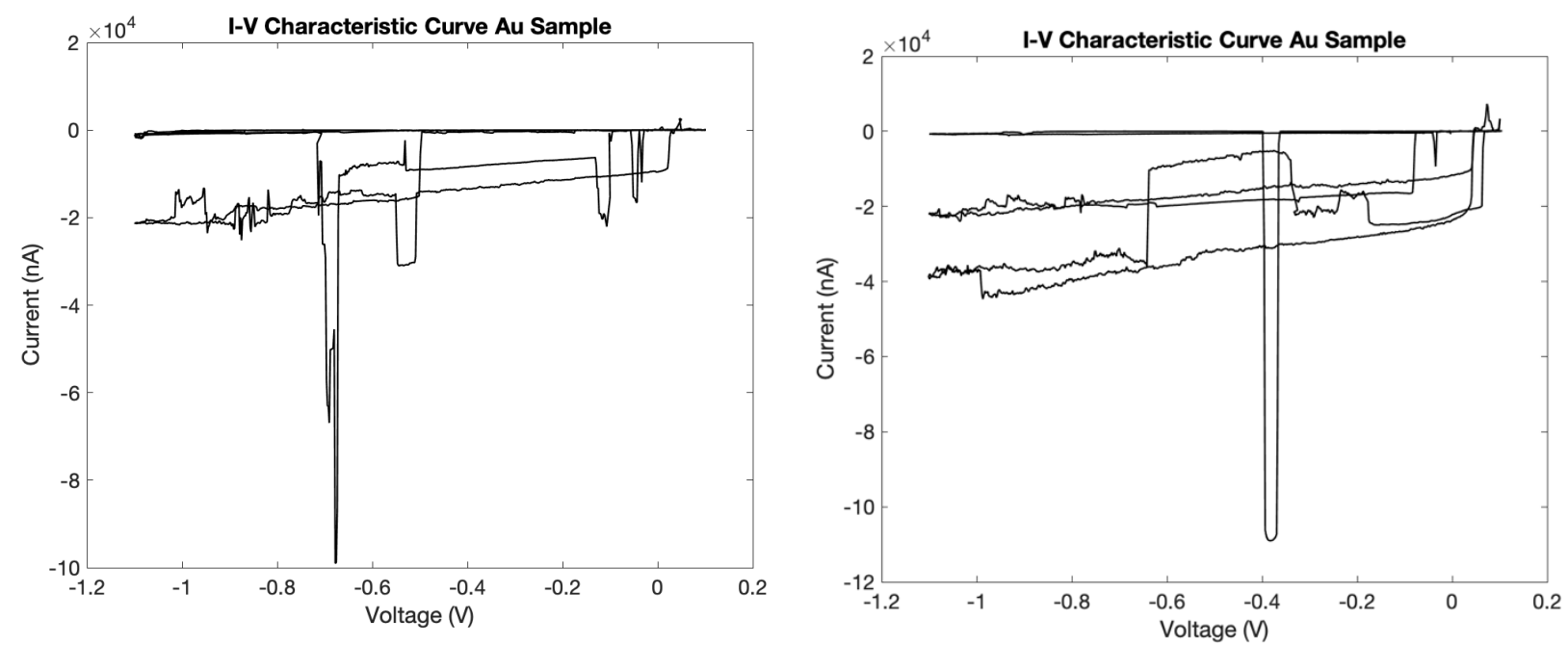

Figure 1. Quantitative I-V measurements taken during cycling without (left) and with (right) full spectrum light provision to the sample. Note the changes in both the onset of I-V excursions and differences in their onset as indicated by the differences in voltage.

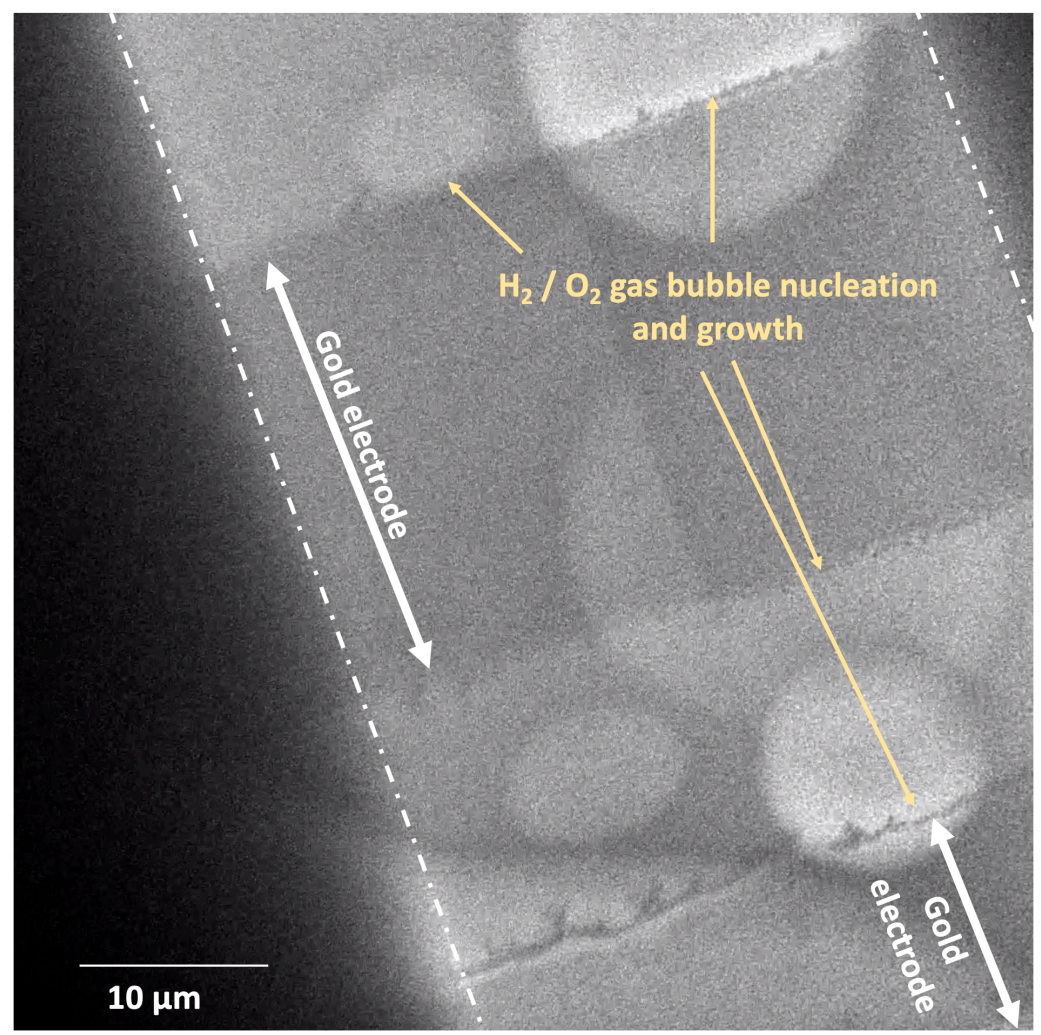

Figure 2. Low-magnification bright field TEM image extracted from a real time video of the splitting of water by Pt electrodes. The silicon nitride viewing window is between the dotted white lines, and the gold electrodes are indicated. The nucleation and growth of gaseous $\mathrm{H}_{2}$ and $\mathrm{O}_{2}$ is visible as indicated. 